\begin{tabular}{|c|l|}
\hline Title & Opening of a pseudogap in a quasi-two-dimensional superconductor due to critical thermal fluctuations \\
\hline Author(s) & Ohkawa, Fusayoshi J. \\
\hline Citation & $\begin{array}{l}\text { Physical Review B, 74(13), 134503 } \\
\text { https://doi.org/10.1103/PhysRevB.74.134503 }\end{array}$ \\
\hline Issue Date & 2006-10 \\
\hline Doc URL & http://hdl.handle.net/2115/16862 \\
\hline Type & article \\
\hline Note & Copyright $\odot 2006$ A merican Physical Society \\
\hline File Information & PRB74.pdf \\
\hline
\end{tabular}

Instructions for use 


\title{
Opening of a pseudogap in a quasi-two-dimensional superconductor due to critical thermal fluctuations
}

\author{
Fusayoshi J. Ohkawa* \\ Department of Physics, Faculty of Science, Hokkaido University, Sapporo 060-0810, Japan \\ (Received 14 August 2005; revised manuscript received 12 June 2006; published 10 October 2006)
}

\begin{abstract}
We examine the role of the anisotropy of superconducting critical thermal fluctuations in the opening of a pseudogap in a quasi-two-dimensional superconductor such as a cuprate-oxide high-temperature superconductor. When the anisotropy between planes and their perpendicular axis is large enough and its superconducting critical temperature $T_{c}$ is high enough, the fluctuations are much developed in the critical region so that lifetime widths of quasiparticles are large and the energy dependence of the self-energy deviates from that of Landau's normal Fermi liquids. A pseudogap opens in such a critical region because quasiparticle spectra around the chemical potential are swept away due to the large lifetime widths. The pseudogap never smoothly evolves into a superconducting gap; it starts to open at a temperature higher than $T_{c}$ while the superconducting gap starts to open just at $T_{c}$. When $T_{c}$ is rather low but the ratio of $\varepsilon_{G}(0) / k_{B} T_{c}$, with $\varepsilon_{G}(0)$ the superconducting gap at $T=0 \mathrm{~K}$, is much larger than a value of about 4 according to the mean-field theory, the pseudogap must be closing as temperature $T$ approaches to the low $T_{c}$ because thermal fluctuations become less developed as $T$ decreases. Critical thermal fluctuations cannot cause the opening of a prominent pseudogap in an almost isotropic three-dimensional superconductor, even if its $T_{c}$ is high.
\end{abstract}

DOI: 10.1103/PhysRevB.74.134503

PACS number(s): 74.20. $-\mathrm{z}, 71.10 .-\mathrm{w}, 74.90 .+\mathrm{n}$

\section{INTRODUCTION}

The elucidation of the mechanism of high criticaltemperature (high- $T_{c}$ ) superconductivity occurring in cuprate-oxide superconductors is an important and long standing issue since its discovery in $1986 .{ }^{1}$ On the other hand, many unconventional normal-state properties are observed: the so-called spin-gap behavior or the reduction of the nuclear magnetic relaxation (NMR) rate with decreasing temperatures $T,{ }^{2}$ the opening of a pseudogap in quasiparticle spectra, ${ }^{3-10}$ and so on. The issue on the mechanism of high$T_{c}$ superconductivity cannot be settled unless not only high$T_{c}$ superconductivity but also such unconventional properties are explained within a theoretical framework. It is widely believed that the reduction of the NMR rate above $T_{c}$ is due to the opening of the pseudogap. It is a key issue to clarify the relation between the pseudogap above $T_{c}$ and a superconducting (SC) gap below $T_{c}$ or whether or not the pseudogap smoothly evolves into the SC gap.

One may argue that the opening of a pseudogap must be a precursor effect of a possible low-temperature instability, antiferromagnetism, superconductivity, or an exotic one. In actual, many possible mechanisms of pseudogaps have already been proposed along these scenarios. ${ }^{11-27}$ Since cuprateoxide superconductors are highly anisotropic quasi-twodimensional ones, it is also a reasonable argument that, even if either of these scenarios is relevant, low dimensionality must play a crucial role. If a second-order phase transition occurred at a nonzero critical temperature $T_{c}$ in one or two dimensions, eventual effects of critical thermal fluctuations or their integrated effects over the wave-number space would diverge at the nonzero $T_{c}$. This leads to a conclusion that no order is possible at nonzero temperatures in one and two dimensions. ${ }^{28}$ It also leads to a speculation that $T_{c}$ of highly anisotropic quasi-low-dimensional superconductors must be substantially reduced by SC critical thermal fluctuations and the reduction of $T_{c}$ must be accompanied by some normalstate anomalies. Since pseudogap structures are certainly substantial in SC critical regions of cuprate-oxide superconductors, it must be clarified first of all how crucial a role SC critical fluctuations can play in the reduction of $T_{c}$ and the opening of pseudogaps, even if any other type of fluctuations or any other mechanism plays a role or a major role.

Critical temperatures $T_{c}$ are reduced by SC critical thermal fluctuations. On the other hand, SC gaps at $T=0 \mathrm{~K}$, $\varepsilon_{G}(0)$, can never be reduced by them because they vanish at $T=0 \mathrm{~K}$. Therefore, large ratios of $\varepsilon_{G}(0) / k_{B} T_{c} \geqslant 8,{ }^{7-9}$ with $k_{B}$ the Boltzmann constant, are pieces of evidence that $T_{c}$ are actually substantially reduced even if observed $T_{c}$ are high. It is plausible that the opening of a pseudogap must be one of normal-state anomalies accompanying the reduction of $T_{c}$.

It has been shown in a previous paper ${ }^{29}$ that when correlation lengths of SC fluctuations are long enough at high enough temperatures in complete two dimensions the renormalization of quasiparticles due to SC fluctuations can cause the opening of a pseudogap. One of the main purposes of this paper is to clarify the role of the anisotropy of SC critical thermal fluctuations in the renormalization of quasiparticles and the opening of a pseudogap in quasi-two-dimensions. When we consider cuprate-oxide superconductors, we should take a repulsive strong-coupling model. However, we consider an attractive intermediate-coupling model ${ }^{13,14}$ in order to demonstrate the essence of a mechanism proposed in this paper. This paper is organized as follows. The formulation is presented in Sec. II. It is demonstrated in Sec. III that a pseudogap can open because of highly anisotropic SC critical thermal fluctuations. It is argued in Sec. IV that the fluctuations must play a role in the opening of pseudogaps in cuprate-oxide superconductors. Discussion is given in Sec. V. Conclusion is presented in Sec. VI. An argument is presented in the Appendix in order to show the relevance of a scenario that high- $T_{c}$ superconductivity of cuprate oxides oc- 
curs in an attractive intermediate-coupling regime for superconductivity, which is realized in a repulsive strong-coupling regime for electron correlations.

\section{FORMULATION}

We consider an attractive intermediate-coupling model on a quasi-two-dimensional lattice composed of square lattices,

$$
\mathcal{H}=\sum_{i j \sigma} t_{i j} a_{i \sigma}^{\dagger} a_{j \sigma}+\frac{1}{2} \sum_{i j \sigma \sigma^{\prime}} U_{i j} a_{i \sigma}^{\dagger} a_{j \sigma^{\prime}}^{\dagger} a_{j \sigma^{\prime}} a_{i \sigma} .
$$

When transfer integrals $t_{i j}$ between nearest and next-nearest neighbors on a plane, $-t$ and $-t^{\prime}$, are considered, the dispersion relation of electrons is given by

$$
E(\mathbf{k})=-2 t\left[\cos \left(k_{x} a\right)+\cos \left(k_{y} a\right)\right]-4 t^{\prime} \cos \left(k_{x} a\right) \cos \left(k_{y} a\right),
$$

with $a$ the lattice constant of square lattices; the bandwidth is $8|t|$. We denote attractive interactions $U_{i j}$ between onsite and nearest-neighbor pairs on a plane by $U_{0}$ and $U_{1}$. We consider two models: (i) $U_{0} /|t| \simeq-4$ and $U_{1}=0$, and (ii) $U_{0}=0$ and $U_{1} /|t| \simeq-4$. In model (i), SC fluctuations corresponding to isotropic $s$-wave superconductivity are developed. In model (ii), SC fluctuations corresponding to anisotropic $s$-wave, $p$-wave, or $d_{x^{2}-y^{2-}}$ or $d \gamma$-wave superconductivity can be developed. We consider only two cases: the isotropic $s$-wave case in model (i) and the $d \gamma$-wave case in model (ii). We consider quasi-two-dimensional features phenomenologically by introducing an anisotropy factor for correlation lengths of SC fluctuations, as is discussed below.

We define a SC susceptibility for singlet superconductivity by ${ }^{30}$

$$
\begin{aligned}
\chi_{\Gamma \Gamma^{\prime}}\left(i \omega_{l}, \mathbf{q}\right)= & \int_{0}^{1 / k_{B} T} d \tau e^{-i \omega_{l} \tau} \frac{1}{N} \sum_{\mathbf{k p}} \eta_{\Gamma}(\mathbf{k}) \eta_{\Gamma^{\prime}}(\mathbf{p}) \\
& \times\left\langle a_{\mathbf{k}+1 / 2 \mathbf{q} \uparrow}(\tau) a_{-\mathbf{k}+1 / 2 \mathbf{q} \downarrow}(\tau) a_{-\mathbf{p}+1 / 2 \mathbf{q} \downarrow}^{\dagger} a_{\mathbf{p}+1 / 2 \mathbf{q} \uparrow}^{\dagger}\right\rangle,
\end{aligned}
$$

where $\omega_{l}=2 l \pi k_{B} T$, with $l$ an integer, is a bosonic energy. Here, $\eta_{\Gamma}(\mathbf{k})$ is a form factor of $\Gamma$-wave Cooper pairs,

$$
\eta_{s}(\mathbf{k})=1 \text {, }
$$

for the isotropic $s$ wave of model (i) and

$$
\eta_{\Gamma}(\mathbf{k})=\left\{\begin{array}{l}
\cos \left(k_{x} a\right)+\cos \left(k_{y} a\right), \quad \Gamma=s, \\
\cos \left(k_{x} a\right)-\cos \left(k_{y} a\right), \quad \Gamma=d \gamma,
\end{array}\right.
$$

for the anisotropic $s$ and $d \gamma$ waves of model (ii). We assume that the conventional condensation of Cooper pairs with zero total momenta occurs below a critical temperature $T_{c}$. When superconductivity of the isotropic $\Gamma=s$ or $\Gamma=d \gamma$ wave occurs, the homogeneous and static part of the SC susceptibility shows a divergence at $T_{c}$. Superconducting $T_{c}$ can be determined from the condition of

$$
\left[\chi_{\Gamma \Gamma}(0,|\mathbf{q}| \rightarrow 0)|t|\right]_{T \rightarrow T_{c}+0} \rightarrow+\infty .
$$

The divergence implies that critical fluctuations can play a role, at least, in SC critical regions of highly anisotropic quasi-two-dimensions.
We divide the self-energy correction into two terms,

$$
\Sigma_{\sigma}\left(i \varepsilon_{n}, \mathbf{k}\right)=\Sigma_{\sigma}^{(\mathrm{SC})}\left(i \varepsilon_{n}, \mathbf{k}\right)+\Sigma_{\sigma}^{\prime}\left(i \varepsilon_{n}, \mathbf{k}\right),
$$

where $\varepsilon_{n}=(2 n+1) k_{B} T$, with $n$ an integer, is a fermionic energy. The first term $\Sigma_{\sigma}^{(\mathrm{SC})}\left(i \varepsilon_{n}, \mathbf{k}\right)$ is due to SC fluctuations of the isotropic $s$ or $d \gamma$ wave and is of linear order in $\chi_{\Gamma \Gamma}\left(i \omega_{l}, \mathbf{q}\right)$, and the second term $\Sigma_{\sigma}^{\prime}\left(i \varepsilon_{n}, \mathbf{k}\right)$ is due to other fluctuations such as SC fluctuations of other waves, charge fluctuations, higher-order terms in SC and charge fluctuations, and so on. When fluctuations of a single wave, the $s$ or $d \gamma$ wave, are considered, ${ }^{30}$ it follows that

$$
\begin{aligned}
\Sigma_{\sigma}^{(\mathrm{SC})}\left(i \varepsilon_{n}, \mathbf{k}\right)= & -\frac{k_{B} T}{N} \sum_{\omega_{l} \mathbf{q}} U_{\Gamma}^{2} \eta_{\Gamma}^{2}\left(\mathbf{k}-\frac{1}{2} \mathbf{q}\right) \chi_{\Gamma \Gamma}\left(i \omega_{l}, \mathbf{q}\right) \\
& \times G_{-\sigma}\left(-i \varepsilon_{n}-i \omega_{l},-\mathbf{k}-\mathbf{q}\right),
\end{aligned}
$$

with $N$ the number of unit cells,

$$
U_{\Gamma}= \begin{cases}U_{0}, & \Gamma=s, \\ U_{1}, & \Gamma=d \gamma,\end{cases}
$$

an effective attractive interaction for the $s$ or $d \gamma$ wave, and

$$
G_{\sigma}\left(i \varepsilon_{n}, \mathbf{k}\right)=\frac{1}{i \varepsilon_{n}+\mu-E(\mathbf{k})-\Sigma_{\sigma}\left(i \varepsilon_{n}, \mathbf{k}\right)},
$$

the renormalized Green function, with $\mu$ the chemical potential. Since we are interested in SC critical fluctuations of $\Gamma$-wave superconductivity, we assume that critical points of other instabilities are a little far way from the critical point or region of $\Gamma$-wave superconductivity. Then, the energy and wave-number dependences of $\Sigma_{\sigma}^{\prime}\left(i \varepsilon_{n}, \mathbf{k}\right)$ can play no significant role in the $\mathrm{SC}$ critical region, so that we simply assume

$$
\Sigma_{\sigma}^{\prime}\left(i \varepsilon_{n}, \mathbf{k}\right)=-i \gamma \frac{\varepsilon_{n}}{\left|\varepsilon_{n}\right|}
$$

Here, $\gamma$ is the lifetime width of quasiparticles due to other fluctuations except for those of the considered $s$-wave or $d \gamma$-wave SC fluctuations. Although it is desirable to calculate self-consistently the total self-energy $\Sigma_{\sigma}\left(i \varepsilon_{n}, \mathbf{k}\right)$ to satisfy Eq. (2.8), ${ }^{31}$ we approximate $G_{\sigma}\left(i \varepsilon_{n}, \mathbf{k}\right)$ in the right-hand side of Eq. (2.8) by an unperturbed one given by

$$
G_{\sigma}^{(0)}\left(i \varepsilon_{n}, \mathbf{k}\right)=\frac{1}{i \varepsilon_{n}+\mu-E(\mathbf{k})-\Sigma_{\sigma}^{\prime}\left(i \varepsilon_{n}, \mathbf{k}\right)} .
$$

Since critical fluctuations are restricted to a narrow region around $\mathbf{q}=0$, Eq. (2.8) is approximately given by

$$
\begin{aligned}
\Sigma_{\sigma}^{(\mathrm{SC})}\left(i \varepsilon_{n}, \mathbf{k}\right)= & -U_{\Gamma}^{2} \eta_{\Gamma}^{2}(\mathbf{k}) k_{B} T \\
& \times \sum_{\omega_{l}} G_{-\sigma}^{(0)}\left(-i \varepsilon_{n}-i \omega_{l}, \mathbf{k}\right) \frac{1}{N} \\
& \times \sum_{\left|\mathbf{q}_{\|}\right| \leqslant q_{c}} \sum_{q_{z}} \chi_{\Gamma \Gamma}\left(i \omega_{l}, \mathbf{q}\right),
\end{aligned}
$$

where the summation over $\mathbf{q}_{\|}=\left(q_{x}, q_{y}\right)$ is restricted to $\left|\mathbf{q}_{\|}\right|$ $\leqslant q_{c}$. The density of states is given by the retarded Green function in such a way that 


$$
\rho(\varepsilon)=\frac{1}{N} \sum_{\mathbf{k}} \rho_{\mathbf{k}}(\varepsilon)
$$

with

$$
\rho_{\mathbf{k}}(\varepsilon)=\left(-\frac{1}{\pi}\right) \operatorname{Im}\left(\frac{1}{\varepsilon+\mu-E(\mathbf{k})+i \gamma-\Sigma_{\sigma}^{(\mathrm{SC})}(\varepsilon+i 0, \mathbf{k})}\right)
$$

being the spectral weight of quasiparticles with energy $\varepsilon$ and wave number $\mathbf{k}$.

When an anisotropic three-dimensional model is considered, it is straightforward to carry out self-consistently a numerical calculation according to the above formulation. Since the anisotropy of SC thermal critical fluctuations plays the most crucial role in the opening of a pseudogap, it is much more convenient to use a phenomenological SC susceptibility, which can explicitly include the anisotropy factor for SC correlation lengths, within the two-dimensional model (2.1) than to use a microscopically derived one for the anisotropic three-dimensional model. The SC retarded susceptibility can be approximately but well described by a phenomenological one,

$$
\chi_{\Gamma \Gamma}(\omega+i 0, \mathbf{q})=\frac{\chi_{\Gamma}(0) \kappa^{2}}{\kappa^{2}+\left(q_{\|} a\right)^{2}+\delta^{2}\left(q_{z} c\right)^{2}+\alpha \omega-i \frac{\omega}{\Gamma_{\mathrm{SC}}|t|}} .
$$

This is similar to the well known one for the spin susceptibility except for the existence of the so-called $\omega$-linear real term, $\alpha \omega$, with $\alpha$ being real. According to a microscopic calculation, such as is carried out in the previous paper, ${ }^{29}$ we can show that $|\alpha| \ll 1$; the $\omega$-linear term is ignored because it plays no significant role. We can also show that Eq. (2.16) can be used not only for $\left|q_{\|} a\right| \ll 1$ and $|\omega / t| \ll 1$ but also for a little larger region than that, that is, at least for $\left|q_{\|} a\right| \leqslant 2$ and $|\omega / t| \lesssim 2$. In Eq. (2.16), $\chi_{\Gamma}(0)$ is the static homogeneous one or $\chi_{\Gamma}(0)=\chi_{\Gamma \Gamma}(0,|\mathbf{q}| \rightarrow 0)$, and $\Gamma_{\mathrm{SC}}|t|$ is an energy scale of SC fluctuations. We introduce no cutoff in the $\omega$ or energy integration of Eq. (2.13) because Eq. (2.13) is never sensitive of the cutoff energy $\omega_{c}$ as long as $\omega_{c} /|t| \geq 2$. In Eq. (2.16), $c$ is introduced for the lattice constant along the $z$ axis, and a factor $\kappa$ and an anisotropy factor $\delta$ are introduced in such a way that SC correlation lengths parallel to the $x-y$ planes and along the $z$ axis are $a / \kappa$ and $\delta c / \kappa$, respectively. We introduce a cutoff wave number $q_{c}=\pi / 3 a$ in the $\mathbf{q}_{\|}$integration of Eq. (2.13); the $\mathbf{q}$ integration is carried out over a region of $\left|\mathbf{q}_{\|}\right| a \leqslant \pi / 3$ and $\left|q_{z}\right| c \leqslant \pi$. According to the definition of the anisotropy factor $\delta$ by Eq. (2.16), the absolute magnitudes of $a$ and $c$, the anisotropy of the lattice constants, or the difference between $a$ and $c$ plays no role in the framework of this paper.

In general, $\quad \chi_{\Gamma}(0)|t| \propto\left[\left(T-T_{c}\right) / T_{c}\right]^{-\lambda} \quad$ and $\quad \kappa^{2} \propto[(T$ $\left.\left.-T_{c}\right) / T_{c}\right]^{\lambda}$ as $T \rightarrow T_{c}+0$, with $\lambda$ being a critical exponent. ${ }^{32}$ Since $\chi_{\Gamma}(0)|t| \kappa^{2}$ is almost independent of $T$ at $T>T_{c}$ in such a way that

$$
\chi_{\Gamma}(0)|t| \kappa^{2} \simeq 1,
$$

we assume $U_{\Gamma} /|t| \simeq-4$ or

$$
g_{\Gamma} \equiv \frac{1}{\pi}\left(U_{\Gamma} / t\right)^{2} \chi_{\Gamma}(0)|t| \kappa^{2}=4,
$$

in both the two cases. We also assume $t^{\prime}=-0.3 t<0$ for transfer integrals and $\mu /|t|=-0.5$ for the chemical potential.

\section{OPENING OF A PSEUDOGAP AT A CRITICAL TEMPERATURE}

In this section, we restrict our examination to the $\mathrm{SC}$ critical temperature, $T=T_{c}$, so that $\kappa=0$. Although $T_{c}$, which is determined from Eq. (2.6), depends on other parameters, we treat it as an independent one. Then, free parameters are $T_{c}$, $\delta, \Gamma_{\mathrm{SC}}$, and $\gamma$. Qualitative features are the same among results for different $\gamma$, unless $\gamma$ are extremely large such as $\gamma /|t| \gg 1$; if a fine structure appears in a physical property it is sharper for a smaller $\gamma .{ }^{33}$ We present here only results for $\gamma /|t|=0.5$, that is, results for $\gamma /|t|=0.5$, and various sets of $T_{c}, \delta$, and $\Gamma_{\mathrm{SC}}$.

Figure 1 shows the imaginary part of the self-energy,

$$
-\operatorname{Im}\left[\Sigma_{\sigma}^{(\mathrm{SC})}(\varepsilon+i 0, \mathbf{k})\right] / g_{\Gamma} \eta_{\Gamma}^{2}(\mathbf{k})|t|,
$$

as a function of $\varepsilon$ for three cases of $E(\mathbf{k})-\mu$; the $\mathbf{k}$ dependence of Eq. (3.1) comes through $E(\mathbf{k})$. When fluctuations are isotropic $(\delta=1)$, the self-energy is small and its $\varepsilon$ dependence is consistent with that of Landau's normal Fermi liquids, as is shown in Fig. 1(d). As long as $T_{c}$ or $T$ is low enough, lifetime widths of quasiparticles are also small and the $\varepsilon$ dependence of the self-energy is also consistent with that of Landau's normal Fermi liquids, even when the anisotropy is large. When fluctuations are anisotropic $(\delta \ll 1)$ and $T_{c}$ or $T$ is high enough, on the other hand, the imaginary part of the self-energy is large and it has a peak around the chemical potential, as is shown in Figs. 1(a)-1(c). The bandwidth of quasiparticles is about $8|t|, \eta_{s}^{2}(\mathbf{k})=1$ for $s$ wave, $\eta_{d \gamma}^{2}( \pm \pi / a, 0)=\eta_{d \gamma}^{2}(0, \pm \pi / a)=4$ for $d \gamma$ wave, and we assume $g_{\Gamma}=4$ for both the waves. Therefore, quasiparticles are not well defined or incoherent on the whole Fermi surface in case of the $s$ wave provided that $-\operatorname{Im}\left[\Sigma_{\sigma}(\varepsilon\right.$ $+i 0, \mathbf{k})] / g_{s} \eta_{s}^{2}(\mathbf{k})|t| \geqq 1$. They are incoherent around $( \pm \pi / a, 0)$ and $(0, \pm \pi / a)$ in case of the $d \gamma$ wave provided that $-\operatorname{Im}\left[\Sigma_{\sigma}(\varepsilon+i 0, \mathbf{k})\right] / g_{d \gamma} \eta_{d \gamma}^{2}(\mathbf{k})|t| \geq 1 / 4$. For example, Fig. 2 shows the spectral weight $\rho_{\mathbf{k}}(\varepsilon=0)$ for $d \gamma$-wave case, which is defined by Eq. (2.15). The spectral weight $\rho_{\mathbf{k}}(\varepsilon$ $=0)$ is large around $\mathbf{k}=( \pm \pi / 2 a, \pm \pi / 2 a)$ so that quasiparticles are rather well defined there, while $\rho_{\mathbf{k}}(\varepsilon=0)$ is small around $\mathbf{k}=( \pm \pi / a, 0)$ and $\mathbf{k}=(0, \pm \pi / a)$ so that quasiparticles are not well defined there.

Since superconductivity can only occur when lifetime widths are small enough, Fig. 1 implies that the reduction of $T_{c}$ is large in a highly anisotropic quasi-two-dimensional superconductor even when its observed $T_{c}$ is high. The reduction of $T_{c}$ must be small in an almost isotropic threedimensional one.

Figures 3 and 4 show the density of states $\rho(\varepsilon)$ for $\kappa=0$ or at the critical point $T=T_{c}$. Large anisotropy of critical fluc- 

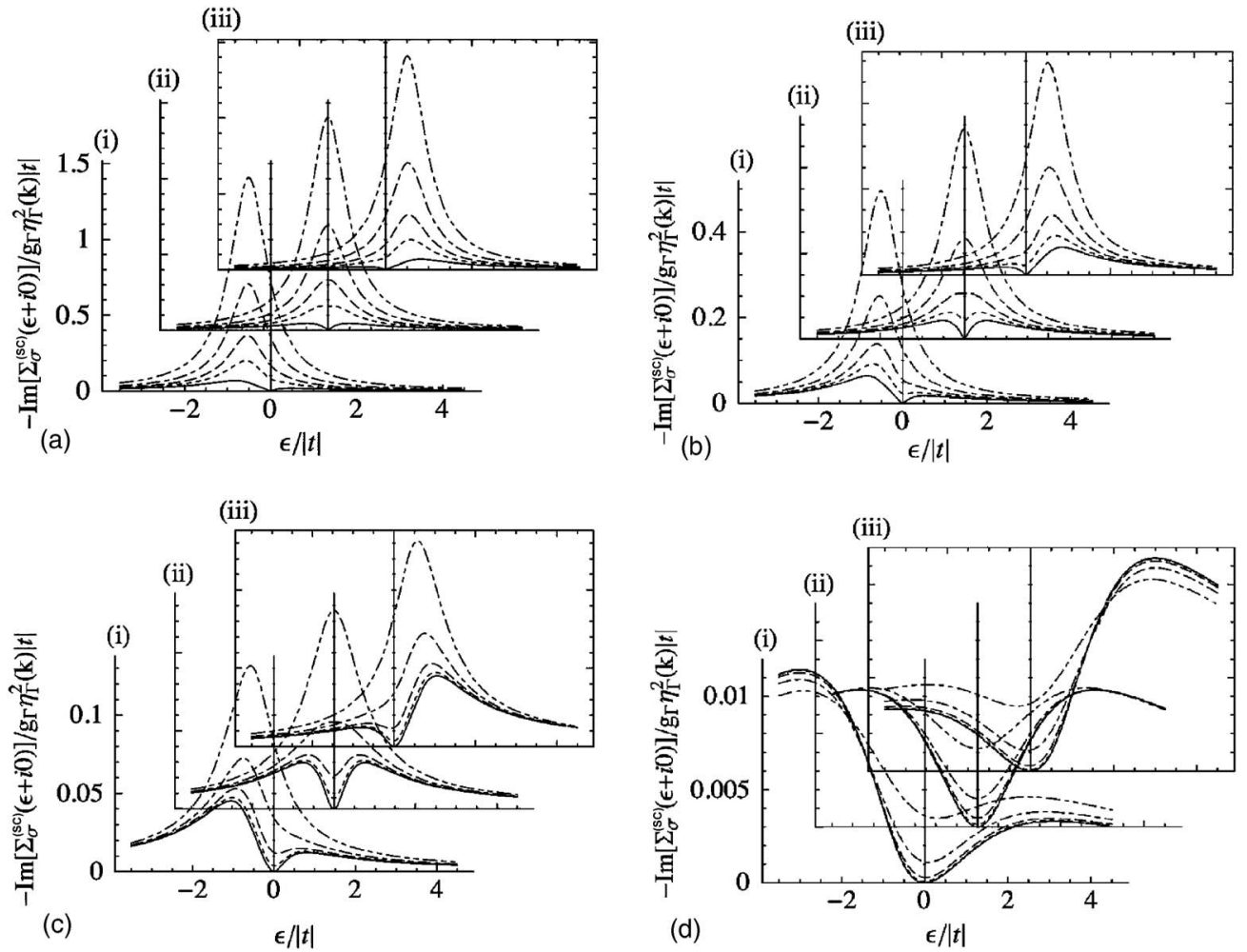

FIG. 1. The imaginary part of the self-energy $\Sigma_{\sigma}^{(\mathrm{SC})}(\varepsilon+i o, \mathbf{k})$ for $\kappa=0, \Gamma_{\mathrm{SC}}=0.2$, and $\gamma /|t|=0.5$ : (a) $\delta=0.01$, (b) $\delta=0.1$, (c) $\delta=0.3$, and (d) $\delta=1$; (i) $E(\mathbf{k})=\mu-0.5|t|$, (ii) $E(\mathbf{k})=\mu$, and (iii) $E(\mathbf{k})=\mu+0.5|t|$. In each figure, solid, dashed, broken, chain, and chain double-dashed lines show results for $k_{B} T_{c} /|t|=0,0.05,0.1,0.2$, and 0.4 , respectively. The imaginary part is larger for a smaller $\delta$ and a higher $T_{c}$. When $\delta$ is small enough and $T_{c}$ is high enough, the $\varepsilon$ dependence is different from that of conventional normal Fermi liquids; there is no minimum at the zero energy or the chemical potential.

tuations or a small $\delta$ such as $\delta<0.1-0.3$ is indispensable for the opening of a prominent pseudogap at $T_{c}$. Smaller energy scales $\Gamma_{\mathrm{SC}}$ or $\Gamma_{\mathrm{SC}}|t|$ are favorable for the opening of pseudogaps. A pseudogap is more prominent for a higher $T_{c}$. Since higher $T_{c}$ are mainly caused by larger $g_{\Gamma}$, this tendency must be larger when the dependence of $T_{c}$ on $g_{\Gamma}$ is consid-

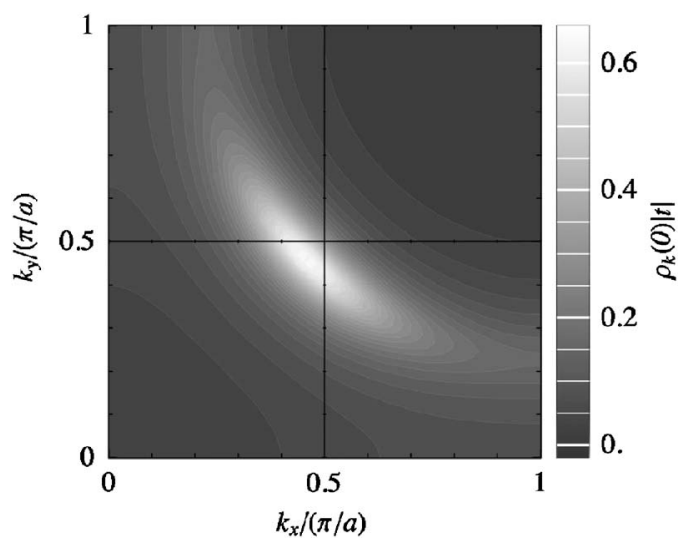

FIG. 2. Spectral weight $\rho_{\mathbf{k}}(\varepsilon=0)$ defined by Eq. (2.15) in onequarter of the two-dimensional Brillouin zone for the $d \gamma$ wave, $\kappa$ $=0, k_{B} T_{c} /|t|=0.2, \delta=0.1, g_{d \gamma}=4, \gamma /|t|=0.5$, and $\mu /|t|=-0.5$. The spectral weight is large around $\mathbf{k}=(\pi / 2 a, \pi / 2 a)$ but is small around $\mathbf{k}=(\pi / a, 0)$ and $\mathbf{k}=(0, \pi / a)$. ered than it is in Figs. 3 and 4. In the isotropic case $(\delta=1)$, on the other hand, a pseudogap structure is absent or subtle at $T_{c}$.

Since a pseudogap opens because quasiparticle spectra around the chemical potential are swept away due to large lifetime widths, the size of the pseudogap is mainly determined from the peak width of the imaginary part of the selfenergy. The peak width is about $2|t|$ for parameters considered in this paper so that the size is also about $2|t|$; the peak width and the size are larger for a higher $T_{c}$ and a smaller $\delta$.

Although spectra of $\rho(\varepsilon)$ are slightly different between the $s$-wave case shown in Fig. 3 and the $d \gamma$-wave case shown in Fig. 4, there is no essential difference between them as long as $U_{s} \simeq U_{d \gamma}$ or $g_{s} \simeq g_{d \gamma}$. The anisotropy of critical fluctuations within planes, the $s$ or $d$ wave, plays a minor role in the opening of pseudogaps.

\section{APPLICATION TO CUPRATE-OXIDE SUPERCONDUCTORS}

When cuprate oxides are considered, the formulation presented in Sec. II should be extended to treat a repulsive strong-coupling regime, $U_{0} /|t| \gtrless 8$ and $\left|U_{1} / t\right| \ll 1$; we should use the so-called $d-p$ or the $t-J$ model. ${ }^{34}$ The most important issue is the nature of single-particle elementary excitations or quasiparticles, which are bound into Cooper pairs, and an 

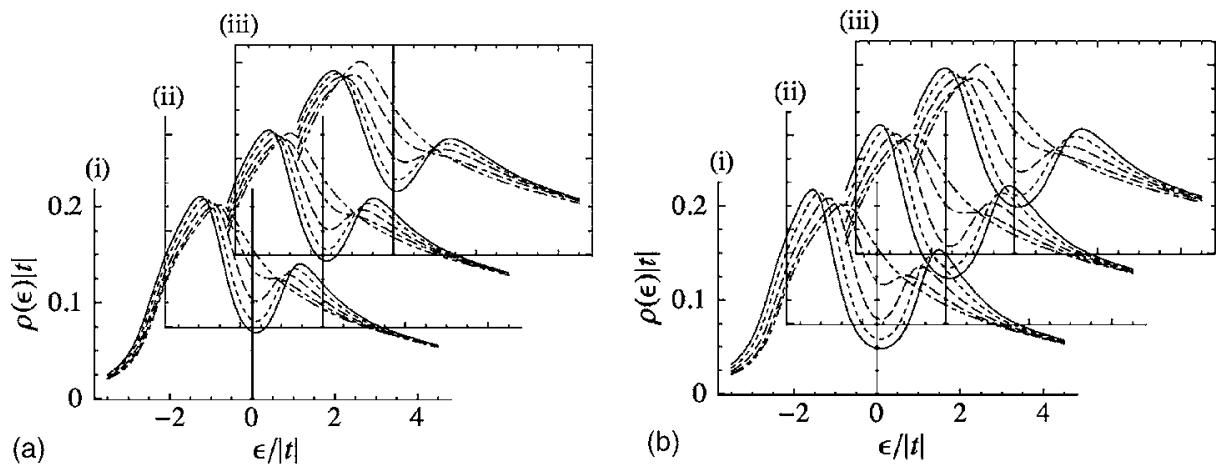

(b)

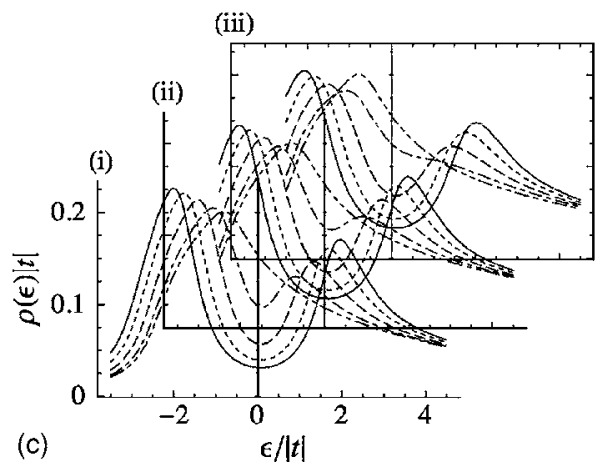

FIG. 3. Density of states $\rho(\varepsilon)$ for the $s$ wave, $\kappa=0, g_{s}=4, \gamma /|t|$ $=0.5$, and $\mu /|t|=-0.5$ : (a) $k_{B} T_{c} /|t|=0.1, \quad(\mathrm{~b}) \quad k_{B} T_{c} /|t|=0.2$, and (c) $k_{B} T_{c} /|t|=0.4$; (i) $\Gamma_{\mathrm{SC}}=0.1$, (ii) $\Gamma_{\mathrm{SC}}=0.3$, and (iii) $\Gamma_{\mathrm{SC}}=1$. In each figure, solid, dashed, broken, chain, and chain double-dashed lines show $\rho(\varepsilon)$ for $\delta=0.01,0.03$, $0.1,0.3$, and 1 , respectively. A pseudogap structure is more prominent for higher $T_{c}$, smaller $\delta$, and smaller $\Gamma_{\mathrm{SC}}$. No prominent one is present in any spectrum for the isotropic case $(\delta=1)$. effective attractive interaction, which works between the quasiparticles. This issue can be solved by a Kondo-lattice theory, as is argued in the Appendix. We present an alternative physical argument on the issue first in this section.

Normal states above $T_{c}$ are unconventional in the socalled under-doped region, as is discussed in the Introduction. However, it is certain that the normal states are Landau's normal Fermi liquids at least in the so-called over-doped region. No phase transition is observed within the normal states above $T_{c}$ as a function of doping concentrations. Ac- cording to the analytical continuation ${ }^{35}$ as a function of doping concentrations, therefore, the normal states above $T_{c}$ must also be Landau's normal Fermi liquids in the whole metallic region even for the so-called under-doped region. The specific heat coefficient of the so-called optimal-doped cuprate oxides is as large as $14 \mathrm{~mJ} / \mathrm{K}^{2} \mathrm{~mol}^{36}$ Then, we can argue with the use of the Fermi-liquid relation ${ }^{37,38}$ that the bandwidth of quasiparticles is as small as $0.3 \mathrm{eV}$ or $|t|$ $\simeq 0.04 \mathrm{eV}$. Although the quasiparticle states are often called midgap states, they correspond to the prediction of Gutzwill-
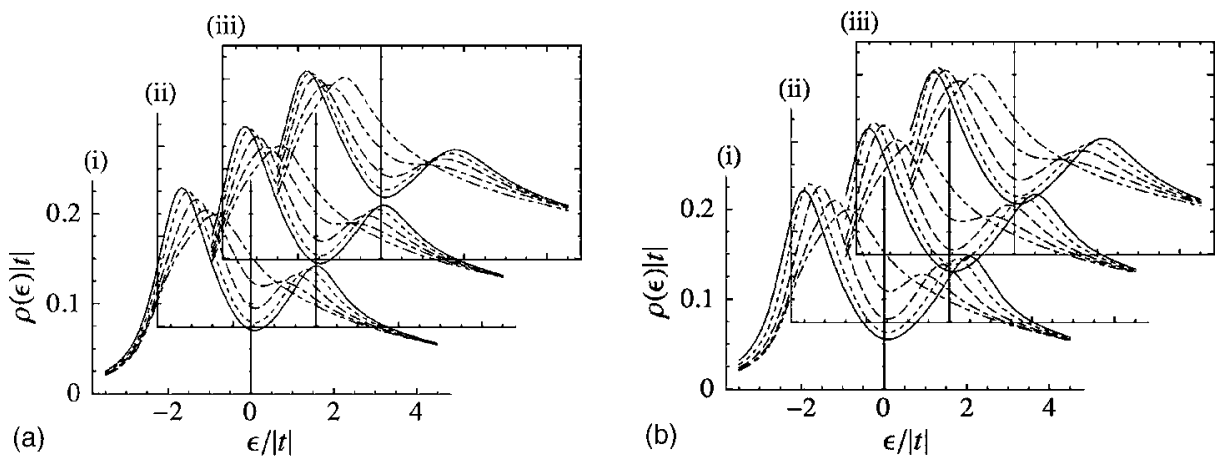

(iii)

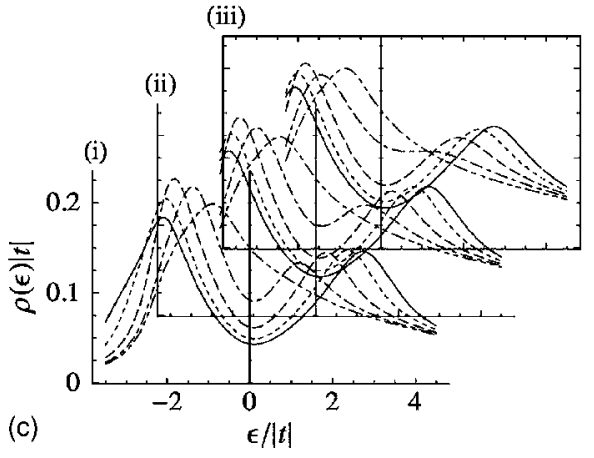

FIG. 4. Density of states $\rho(\varepsilon)$ for the $d \gamma$ wave, $\kappa=0, g_{d \gamma}=4$, $\gamma /|t|=0.5$, and $\mu /|t|=-0.5$. See also the figure caption of Fig. 3 . No essential difference can be seen between Fig. 3 for the $s$ wave and this figure for the $d \gamma$ wave. 

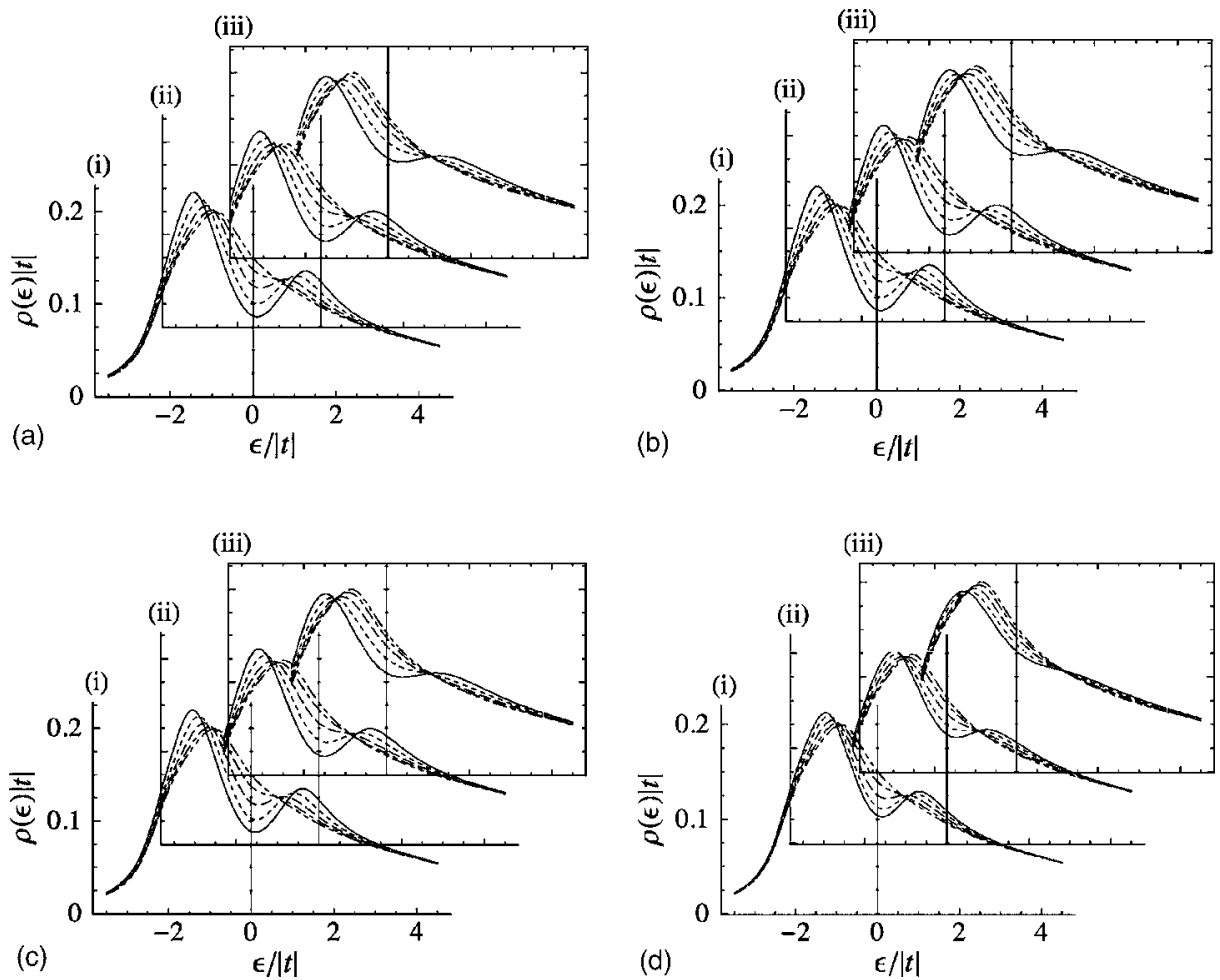

FIG. 5. $\rho(\varepsilon)$ at $k_{B} T /|t|=0.4$ for the $d \gamma$ wave, $g_{d \gamma}=4, \gamma /|t|=0.5$, and $\mu /|t|=-0.5$ : (a) $\delta=0.01$, (b) $\delta=0.03$, (c) $\delta=0.1$, and (d) $\delta$ $=0.3$; (i) $\Gamma_{\mathrm{SC}}=0.1$, (ii) $\Gamma_{\mathrm{SC}}=0.3$, and (iii) $\Gamma_{\mathrm{SC}}=1$. In each figure, solid, dashed, broken, chain, and chain double-dashed lines show $\rho(\varepsilon)$ for $\kappa^{2}=0.5,1,2,4$, and 8 , respectively. When either or both of $\kappa^{2}$ and $\Gamma_{\mathrm{SC}}$ are large enough, a pseudogap structure is absent or subtle. er's theory; ${ }^{39,40}$ we call the quasiparticles Gutzwiller's quasiparticles in this paper. An intersite magnetic exchange interaction can be an attractive interaction to form Cooper pairs. ${ }^{41}$ The main part of the exchange interaction in cuprate oxides is the superexchange interaction. It is antiferromagnetic and is as large as $J_{s}=-0.15 \mathrm{eV}$. It has already been shown in 1987 that high- $T_{c}$ superconductivity can occur when Gutzwiller's quasiparticles are bound into $d \gamma$-wave Cooper pairs due to the superexchange interaction. ${ }^{42}$ According to this scenario, high- $T_{c}$ superconductivity occurs in an attractive intermediate-coupling regime $\left|J_{s} / t\right| \simeq 4$ for superconductivity, which is realized in the repulsive strongcoupling regime for electron correlations. The Kondo-lattice theory, as is briefly argued in the Appendix, is consistent with this physical argument based on the analytical continuation.

Since $k_{B} T_{c} /|t|=0.2$ corresponds to $T_{c} \simeq 100 \mathrm{~K}$ and $\delta$ must be as small as $\delta \leqq 0.1$ in cuprate oxides, Fig. 4(b) implies that the opening of a pseudogap at $T_{c}$ must be mainly due to SC critical thermal fluctuations. It is plausible that even if other mechanisms work the fluctuations play a major role in the opening of pseudogaps, at least, at $T_{c}$ and in SC critical regions of high- $T_{c}$ cuprate-oxide superconductors.

If all the parameters such as $\kappa, \delta$, and $\Gamma_{\mathrm{SC}}$, were constant as a function of $T$, pseudogaps would be developed with increasing $T$, as is shown in Figs. 3 and 4. Experimentally, however, pseudogaps close at high enough $T$. It is likely that the temperature dependences of $\kappa, \delta$, and $\Gamma_{\mathrm{SC}}$ are responsible for the closing of pseudogaps, for example, at $k_{B} T /|t| \simeq 0.4$ or $T \simeq 200 \mathrm{~K}$. Then, we examine what conditions are needed for the parameters to exhibit that a pseudogap that opens at $k_{B} T /|t|=0.2$ closes at $k_{B} T /|t|=0.4$. It is obvious that $\kappa^{2}=0$ at $T=T_{c}$ and $\kappa^{2}>0$ at $T>T_{c}$ or that $\kappa^{2}$ increases with increasing $T ; \chi_{\Gamma}(0) \kappa^{2}$ is almost constant, as is shown in Eq. (2.17).
It is also obvious that $\Gamma_{\mathrm{SC}}$ also increases with increasing $T$. Figure 5 shows that when either or both of $\kappa^{2}$ and $\Gamma_{\mathrm{SC}}$ are large enough no prominent pseudogap can be seen at $k_{B} T /|t|=0.4$. It is interesting to complete the self-consistent procedure, ${ }^{31}$ where the SC susceptibility is microscopically calculated and Eq. (2.8) is used instead of Eq. (2.13), in order to confirm whether or not such temperature dependences of $\kappa$ and $\Gamma_{\mathrm{SC}}$ can be actually reproduced.

\section{DISCUSSION}

It is desirable that the theoretical framework of this paper should be self-consistently completed. However, the selfconsistent procedure depends on microscopic physical processes or on what effective Hamiltonian is used, an intermediate-coupling attractive model or a strong-coupling repulsive model. ${ }^{31}$ One of the reasons why we take a phenomenological treatment in this paper is to demonstrate the essence of the proposed mechanism on pseudogaps due to thermal SC critical fluctuations, which does not depend on microscopic models.

According to the mean-field theory for $d \gamma$-wave superconductivity, ${ }^{42}$

$$
\varepsilon_{G}(0) / k_{B} T_{c} \simeq 4.35,
$$

with $\varepsilon_{G}(0)$ the superconducting gap at $T=0 \mathrm{~K}$. Since SC thermal fluctuations vanish at $T=0 \mathrm{~K}$, the reduction of $\varepsilon_{G}(0)$ must be very small. As is discussed in Introduction, therefore, observed large ratios ${ }^{7-9}$ of

$$
\varepsilon_{G}(0) / k_{B} T_{c} \geq 8,
$$

are pieces of evidence that $T_{c}$ are actually reduced by the thermal fluctuations, at least, in optimal-doped or moderately 
under-doped cuprate oxides, where $T_{c}$ are rather high. Critical thermal fluctuations must play a major role in the opening of pseudogaps in such cuprate-oxide superconductors with rather high $T_{c}$.

Since SC thermal fluctuations vanish at $T=0 \mathrm{~K}$, we expect that a pseudogap due to the thermal fluctuations is closing as $T \rightarrow T_{c}$ in complete two dimensions, where $T_{c}$ is definitely zero. A similar argument applies to quasi-twodimensions, where $T_{c}$ can be nonzero. A SC gap starts to open at nonzero $T_{c}$ and a pseudogap starts to open at $T$ a little higher than $T_{c}$. When $T_{c}$ is low but $\varepsilon_{G}(0) / k_{B} T_{c}$ is large, it is plausible that a pseudogap opens at rather high temperatures and it is closing as $T$ approaches the low- $T_{c}$. The pseudogap never smoothly evolves into the SC gap. It is interesting to examine whether or not pseudogaps are actually closing as $T \rightarrow T_{c}+0$ in under-doped cuprate oxides.

Critical thermal fluctuations cannot play any significant role in the opening of a pseudogap in an almost isotropic three-dimensional superconductor, even if it is of an intermediate coupling for superconductivity so that its $T_{c}$ is high. If a prominent pseudogap opens in an almost isotropic superconductor, a mechanism or mechanisms different from the one proposed in this paper must be responsible for the opening of the pseudogap.

Mercury-based cuprate oxides show very high- $T_{c}$ under pressures. ${ }^{43,44}$ Pressures must reduce the anisotropy so that the reduction of $T_{c}$ becomes smaller with increasing pressures. It is interesting to search for almost isotropic cuprate oxide superconductors with no prominent pseudogap. Since the reduction of $T_{c}$ by critical fluctuations is small, their $T_{c}$ can be higher than $T_{c}$ of quasi-two-dimensional ones. A simple argument implies that if $\varepsilon_{G}(0) / k_{B} T_{c}=4-5$ are realized $T_{c}$ can exceed $200 \mathrm{~K}$.

Transition-metal dichalcogenide and organic superconductors are also low-dimensional superconductors. ${ }^{45,46}$ If $T_{c}$ are high enough and $\varepsilon_{G}(0) / k_{B} T_{c}$ are large enough, pseudogaps must also open in critical regions.

The opening of pseudogaps is also expected in quasi-onedimension. It is interesting to examine effects of not only thermal fluctuations but also quantum fluctuations.

It is straightforward to extend the theory of this paper to pseudogaps due to spin and charge fluctuations. When $T_{c}$ of a spin density wave (SDW) or a charge density wave (CDW) is high enough and the anisotropy of SDW or CDW fluctuations is large enough, a pseudogap must also open in a critical region of SDW or CDW. Conventional SC fluctuations are developed around the zone center, while SDW and CDW ones are developed around wave numbers corresponding to the nesting of the Fermi surface. Scatterings by conventional $\mathrm{SC}$ ones are forward scatterings so that their contribution to the transport relaxation rate is small, while those by SDW and CDW ones must contribute to the transport relaxation rate. It is likely that resistivity is relatively larger in SDW and CDW cases than it is in conventional SC cases.

Critical temperatures $T_{c}$ of under-doped cuprate oxides, which are close to an antiferromagnetic insulating phase, are very low or vanishing. The vanishment of $T_{c}$ can never be explained only in terms of the reduction of $T_{c}$ due to the thermal fluctuations because they vanish at $T=0 \mathrm{~K}$. Other reduction effects of $T_{c}$ such as those due to disorder, SDW or antiferromagnetism, and so on must be considered to explain the vanishment of $T_{c}$.

The so-called zero-temperature pseudogap (ZTPG) is observed at very low temperatures in under-doped cuprates. ${ }^{47,48}$ Thermal critical fluctuations can never explain ZTPG because their effects are small at low temperatures. A mechanism of ZTPG is proposed in a previous paper. ${ }^{49}$ As is discussed in the Appendix, magnetic exchange interactions are responsible for superconductivity as well as magnetism in cuprate oxide superconductors. Then, the competition or an interplay between superconductivity and antiferromagnetism or SDW can play a crucial role. The ZTPG phase must never be a normal Fermi-liquid phase, but it must be a non-Fermi liquid phase where SC and SDW order parameters coexist. Experimentally, antiferromagnetic spin fluctuations are well developed in under-doped cuprates. Disorder or large lifetime widths of quasiparticles due to disorder can play a role in the stabilization of SDW. ${ }^{50}$ The Brillouin zone is folded by the SDW. Then, the condensation of Cooper pairs between two quasiparticles around one of the edges of the folded Brillouin zone or Cooper pairs whose total momenta are $\pm 2 m \mathbf{Q}_{\text {SDW }}$, with $\mathbf{Q}_{\text {SDW }}$ being a wave number of SDW and $m$ being an integer such as $m=1,2,3$, and so on, can occur in addition to that of conventional Cooper pairs with zero total momenta. A 4a-period stripe structure can arise from an $8 a$-period single-Q SDW; a $4 a \times 4 a$ checkerboard structure can arise from a double-Q SDW; a fine structure similar to that of ZTPG can arise from the coexistence of the single- $\mathbf{Q}$ or double-Q SDW and a multi-Q pair density wave of $d \gamma$-wave Cooper pairs. ${ }^{49}$ On the other hand, the normal phase above $T_{c}$ has no phase boundary between under-doped and over-doped regions. Then, the examination of this paper implies that a pseudogap due to thermal SC and SDW critical fluctuations can open in the normal phase of under-doped cuprates where ZTPG and the checkerboard structure are present below $T_{c}$.

\section{CONCLUSION}

We study the role of the anisotropy of superconducting critical thermal fluctuations in the opening of a pseudogap in a quasi-two-dimensional superconductor. The thermal fluctuations are developed in a critical region provided that the anisotropy is large enough and the critical region is extended to high enough temperatures. A large ratio of $\varepsilon_{G}(0) / k_{B} T_{c}$, with $\varepsilon_{G}(0)$ being the superconducting gap at $T=0 \mathrm{~K}$, is a piece of evidence of well-developed thermal fluctuations; thermal fluctuations can reduce $T_{c}$ while they can never reduce $\varepsilon_{G}(0)$, which is for $T=0 \mathrm{~K}$. The well-developed fluctuations make lifetime widths of quasiparticles large. A pseudogap can open because quasiparticle spectra around the chemical potential are swept away due to the large lifetime widths. It can open in a critical region of not only anisotropic superconductivity such as the $d \gamma$-wave one but also isotropic $s$-wave or BCS one. Even if $T_{c}$ is low in a quasi-twodimensional superconductor, a pseudogap can also open at temperatures $T$ substantially higher than $T_{c}$ provided that $\varepsilon_{G}(0) / k_{B} T_{c}$ is large enough. Since thermal fluctuations are vanishing as $T \rightarrow 0 \mathrm{~K}$, the pseudogap of such a low- $T_{c}$ super- 
conductor must be closing as $T \rightarrow T_{c}+0$. Since a pseudogap starts to open at a temperature higher than $T_{c}$ while a superconducting gap starts to open just at $T_{c}$, it never smoothly evolves into the superconducting gap. On the other hand, critical thermal fluctuations cannot cause the opening of a prominent pseudogap in an almost isotropic threedimensional superconductor, even if its $T_{c}$ is high.

Superconducting critical thermal fluctuations must play a major role in the opening of pseudogaps in critical regions of cuprate-oxide superconductors with $\varepsilon_{G}(0) / k_{B} T_{c} \geqslant 8$, even if other mechanisms work there. It is interesting to confirm that pseudogaps above $T_{c}$ never smoothly evolve into superconducting gaps below $T_{c}$ in cuprate-oxide superconductors.

\section{ACKNOWLEDGMENTS}

The author is thankful for useful discussions with M. Ido, M. Oda, and N. Momono.

\section{APPENDIX: QUASIPARTICLES AND ATTRACTIVE INTERACTIONS IN CUPRATE-OXIDE SUPERCONDUCTORS}

We consider one of effective Hamiltonians where the onsite $U$ repulsion plays a crucial role, such as the Hubbard model, the periodic Anderson model, the $d-p$ model, the $t-J$ or $t$-J-infinite $U$ model, ${ }^{51}$ and so on; the formulation and argument of this appendix can be extended almost in parallel to such various on-site $U$ models. We assume the repulsive strong-coupling regime for electron correlations or we assume that the on-site $U$ is as large as the bandwidth of unrenormalized electrons or is larger than it.

According to Hubbard's theory, ${ }^{52,53}$ a band splits into two subbands called the lower Hubbard band (LHB) and the upper Hubbard band (UHB). According to Gutzwiller's theory, ${ }^{39,40}$ with the use of the Fermi-liquid theory, ${ }^{37,38}$ a narrow band of quasiparticles appears around the chemical potential; we call them Gutzwiller's band and Gutzwiller's quasiparticles. The combination of the two theories implies that the density of states must be of a three-peak structure, Gutzwiller's band between LHB and UHB. Actually the formation of Gutzwiller's band at the top of LHB in less-thanhalf filling cases is demonstrated in a previous paper. ${ }^{54}$ The Mott-Hubbard splitting occurs in both metallic and insulating phases, and Gutzwiller's band or quasiparticles are responsible for metallic behaviors.

Any mutual interaction arises from the virtual exchange of a boson, a bosonic excitation, or a bosonic resonance state. For example, Yukawa's nuclear force arises from that of a pion, electromagnetic force from that of a photon, the attractive interaction in conventional BCS superconductors from that of a phonon, and so on. According to this theoretical framework, the superexchange interaction arises from that of a pair excitation of electrons across LHB and UHB; ${ }^{55}$ it is phenomenologically given in the $t-J$ model. It works even in metallic phases as long as the Mott-Hubbard splitting is significant. The virtual exchange of a pair excitation of Gutzwiller's quasiparticles plays no role in the arising of the superexchange interaction, but another exchange interaction arises from it. ${ }^{55}$ In this paper, the exchange interaction is denoted by $J_{Q}\left(i \omega_{l}, \mathbf{q}\right)$. It has an interesting property that its strength is proportional to the width of Gutzwiller's band. Gutzwiller's quasiparticles are bound into Cooper pairs mainly due to the two exchange interactions. The superexchange interaction is antiferromagnetic for any filling, ${ }^{56}$ so that it is attractive for singlet Cooper pairs such as $d \gamma$-wave ones. The exchange interaction $J_{Q}\left(i \omega_{l}, \mathbf{q}\right)$ is antiferromagnetic when the chemical potential lies around the center of Gutzwiller's quasiparticle band, while it is ferromagnetic when the chemical potential lies around the upper or lower edge of Gutzwiller's quasiparticle band. ${ }^{57}$ For singlet Cooper pairs, in general, the exchange interaction $J_{Q}\left(i \omega_{l}, \mathbf{q}\right)$ is attractive in the case of almost half-fillings while it is repulsive far away from half-fillings.

Strong local correlations, which give rise to the threepeak structure, and the exchange interactions can be treated by a Kondo-lattice theory. ${ }^{29,50,55,58}$ The starting point of the Kondo-lattice theory is the single-site approximation (SSA) that includes all the single-site terms. The SSA is reduced to solving the Anderson model self-consistently; ${ }^{59-61}$ any single-site term of the lattice model, one of the effective Hamiltonians with large enough on-site $U$, is equal to its corresponding term of the Anderson model. The local Kondo temperature $T_{K}$ or $k_{B} T_{K}$ is defined as an energy scale of local quantum spin fluctuations of the lattice model. The threepeak structure corresponds to the so-called Kondo peak between two subpeaks in the Anderson model; the width of Gutzwiller's band is about $4 k_{B} T_{K}$.

We define an intersite exchange interaction $I_{s}\left(i \omega_{l}, \mathbf{q}\right)$ by following a physical picture for Kondo lattices that local spin fluctuations at different sites interact with each other by an intersite exchange interaction,

$$
\chi_{s}(\omega+i 0, \mathbf{q})=\frac{\widetilde{\chi}_{s}\left(i \omega_{l}\right)}{1-\frac{1}{4} I_{s}\left(i \omega_{l}, \mathbf{q}\right) \widetilde{\chi}_{s}\left(i \omega_{l}\right)},
$$

with $\chi_{s}\left(i \omega_{l}, \mathbf{q}\right)$ being the spin susceptibility of the lattice model and $\widetilde{\chi}_{s}\left(i \omega_{l}\right)$ being that of the Anderson model. Gutzwiller's quasiparticles are well defined at $T \leqq T_{K}$. The main part of the exchange interaction defined by Eq. (A1) is composed of the exchange interaction $J_{Q}\left(i \omega_{l}, \mathbf{q}\right)$ in addition to the superexchange interaction $J_{s}(\mathbf{q})$,

$$
I_{s}\left(i \omega_{l}, \mathbf{q}\right)=J_{s}(\mathbf{q})+J_{Q}\left(i \omega_{l}, \mathbf{q}\right)-4 \Lambda\left(i \omega_{l}, \mathbf{q}\right) .
$$

The third term $-4 \Lambda\left(i \omega_{l}, \mathbf{q}\right)$ includes mode-mode coupling terms among intersite spin fluctuations, which correspond to those considered in the self-consistent renormalization (SCR) theory of spin fluctuations. ${ }^{62}$ Magnetism at $T \leqslant T_{K}$ is characterized as itinerant-electron one. The mode-mode coupling term ${ }^{62}$ or the exchange interaction ${ }^{63,64} J_{Q}\left(i \omega_{l}, \mathbf{q}\right)$ is responsible for the Curie-Weiss (CW) law of itinerant-electron magnetism; which is responsible for the $\mathrm{CW}$ law depends on the dispersion relation of quasiparticles ${ }^{65}$ On the other hand, Gutzwiller's quasiparticles are never well defined at $T \gg T_{K}$; the exchange interaction $J_{Q}\left(i \omega_{l}, \mathbf{q}\right)$ vanishes. ${ }^{66}$ Magnetism at $T \gg T_{K}$ is characterized as the local-moment one. The local term $\widetilde{\chi}_{s}(0)$ in Eq. (A1) is responsible for the CW law of local-moment magnetism. The Kondo-lattice theory can treat 
not only itinerant-electron magnetism but also local-moment magnetism; it can treat a crossover between them..$^{50}$

The self-energy in the SSA is expanded at $T \lesssim T_{K}$ in such a way that

$$
\widetilde{\Sigma}_{\sigma}\left(i \varepsilon_{n}\right)=\widetilde{\Sigma}_{0}+\left(1-\widetilde{\phi}_{\gamma}\right) i \varepsilon_{n}+\left(1-\widetilde{\phi}_{s}\right) \frac{1}{2} \sigma g \mu_{B} H+\cdots,
$$

with $g$ being the $g$ factor, $\mu_{B}$ the Bohr magneton, and $H$ an infinitesimally small magnetic field. According to the Ward relation, ${ }^{67}$ the irreducible single-site three-point vertex function in spin channels, $\widetilde{\lambda}_{s}\left(i \varepsilon_{n}, i \varepsilon_{n}+i \omega_{l} ; i \omega_{l}\right)$, is given by ${ }^{68}$

$$
\tilde{\lambda}_{s}\left(i \varepsilon_{n}, i \varepsilon_{n}+i \omega_{l} ; i \omega_{l}\right)=\frac{2 \widetilde{\phi}_{s}}{U \tilde{\chi}_{s}\left(i \omega_{l}\right)}\left[1+O\left(\frac{1}{U \tilde{\chi}_{s}\left(i \omega_{l}\right)}\right)\right],
$$

for $\left|\varepsilon_{n}\right| \rightarrow+0$ and $\left|\omega_{l}\right| \rightarrow+0$. When we approximately use Eq. (A4), with higher-order terms in $1 / U \widetilde{\chi}_{s}\left(i \omega_{l}\right)$ being ignored, for small enough $\left|\varepsilon_{n}\right|$ and $\left|\omega_{l}\right|$, it follows that

$$
\frac{1}{4} U^{2} \widetilde{\lambda}_{s}^{2}\left(i \varepsilon_{n}, i \varepsilon_{n}+i \omega_{l} ; i \omega_{l}\right)\left[\chi_{s}\left(i \omega_{l}, \mathbf{q}\right)-\widetilde{\chi}_{s}\left(i \omega_{l}\right)\right]=\widetilde{\phi}_{s}^{2} \frac{1}{4} I_{\mathrm{s}}^{*}\left(i \omega_{l}, \mathbf{q}\right),
$$

with

$$
I_{s}^{*}\left(i \omega_{l}, \mathbf{q}\right)=\frac{I_{s}\left(i \omega_{l}, \mathbf{q}\right)}{1-\frac{1}{4} I_{s}\left(i \omega_{l}, \mathbf{q}\right) \tilde{\chi}_{s}\left(i \omega_{l}\right)} .
$$

The left-hand side is the mutual interaction due to spin fluctuations; the single-site term is subtracted because it is considered in the SSA. The exchange interaction $I_{s}^{*}\left(i \omega_{l}, \mathbf{q}\right)$ is nothing but an exchange interaction enhanced by spin fluctuations. The right-hand side is that due to the enhanced exchange interaction; $\widetilde{\phi}_{s}$ appear as effective single-site vertex functions. The two mechanisms of attractive interactions to form Cooper pairs, the so-called spin-fluctuation mechanism and the exchange-interaction mechanism, are essentially the same as each other. ${ }^{69}$

A starting or unperturbed state is constructed in the SSA; it is definitely a normal Fermi liquid. Then, intersite effects can be perturbatively considered in terms of $I_{s}\left(i \omega_{l}, \mathbf{q}\right)$ or $I_{s}^{*}\left(i \omega_{l}, \mathbf{q}\right)$. Since this formulation, which is a perturbative theory starting from the unperturbed state constructed in the nonperturbative SSA theory, is consistent with the physical picture for Kondo lattices, we should call it a Kondo-lattice theory. Since the SSA is rigorous for paramagnetic phases with no order parameter in infinite dimensions ${ }^{70}$ the perturbative theory can also be formulated as a $1 / d$-expansion theory, with $d$ the spatial dimensionality. The SSA can also be formulated as the dynamical mean-field theory (DMFT) ${ }^{71}$ or the dynamical coherent potential approximation (DCPA). ${ }^{72}$ Leading order effects in $1 / d$ are not only local correlations considered in the SSA, which correspond to dynamical mean fields considered in DMFT, the dynamical coherent potential considered in DCPA, and single-site terms or local effects related with them, but also conventional Weiss's mean fields of certain instabilities. ${ }^{73}$ All the other effects or terms are of higher order in $1 / d$. Not only the Weiss's mean fields but also higher order effects in $1 / d$ can be perturbatively considered.

Taking the Kondo-lattice theory, we can develop a theory of superconductivity occurring in the vicinity of the MottHubbard transition almost in parallel to that of this paper. ${ }^{29,42,50,58}$ Effectively or eventually, $t$ and $t^{\prime}$ of this paper are replaced by those of Gutzwiller's quasiparticles. The onsite part of the eventual mutual interaction is definitely strongly repulsive. Therefore, $T_{c}$ of $s$ wave or BCS superconductivity cannot be high. On the other hand, it plays no role in the effective coupling constant of $d \gamma$-wave superconductivity. The attractive interaction given by Eq. (A5) includes not only the superexchange interaction and the exchange interaction $J_{Q}\left(i \omega_{l}, \mathbf{q}\right)$ themselves but also their enhanced ones, which include effects due to the nesting of the Fermi surface, the so-called internodal scatterings in the $d \gamma$-wave case, and so on. Although it works between not only nearest-neighbor sites but also neighboring sites, its nearest-neighbor part play a major role, at least, provided that the system is a little far away from the critical point of antiferromagnetism. The attractive interaction of this paper is replaced by the nearestneighbor component of $I_{s}^{*}\left(i \omega_{l}, \mathbf{q}\right)$; we simply denote an averaged one over its low-energy part by $I_{s}^{*}$. When the unperturbed state is constructed in the SSA, $U_{d \gamma}$ for the $d \gamma$ wave is replaced by

$$
U_{d \gamma} \rightarrow \widetilde{U}_{d \gamma}^{*}=\frac{3}{4} I_{s}^{*}\left(\widetilde{\phi}_{s} / \widetilde{\phi}_{\gamma}\right)^{2},
$$

where the factor 3 is due to the three spin channels. However, Gutzwiller's quasiparticles constructed in the SSA are further renormalized by SC and antiferromagnetic spin fluctuations. We should use the mass renormalization factor $\phi_{\gamma}(\mathbf{k})$, which includes such an intersite renormalization in addition to the single-site renormalization, instead of $\widetilde{\phi}_{\gamma}$. Then, we should take

$$
U_{d \gamma}^{*}=\frac{3}{4} I_{s}^{*}\left[\widetilde{\phi}_{s} /\left\langle\phi_{\gamma}(\mathbf{k})\right\rangle\right]^{2},
$$

with $\left\langle\phi_{\gamma}(\mathbf{k})\right\rangle$ an average over the Fermi surface.

When we consider cuprate-oxide superconductors, we should use the $d-p$ or $t-J$ model $^{34}$ rather than the Hubbard model. ${ }^{74}$ In the SSA, it follows that $\widetilde{\phi}_{s} / \widetilde{\phi}_{\gamma} \simeq 2$ for almost half-fillings so that theoretical $T_{c}$ are too high to explain observed $T_{c}{ }^{42}$ When we use Eq. (A8) with $\widetilde{\phi}_{s} /\left\langle\phi_{\gamma}(\mathbf{k})\right\rangle$ $\simeq 0.7-1$ instead of Eq. (A7) with $\widetilde{\phi}_{s} / \widetilde{\phi}_{\gamma} \simeq 2$, we can explain observed $T_{c}$.

This appendix can be concluded in the following way. The unperturbed state in the Kondo-lattice theory is definitely a normal Fermi liquid. Since the assumption of the analytical continuation is nothing but assuming that the normal state above $T_{c}$ is a normal Fermi liquid, the Kondolattice theory justifies the assumption of the analytical continuation in Sec. IV. Then, we can examine an instability of the normal Fermi liquid or a symmetry breaking such as a superconducting one caused by conventional Weiss's mean 
fields due to the intersite magnetic exchange interaction $I_{s}\left(i \omega_{l}, \mathbf{q}\right)$ or $I_{s}^{*}\left(i \omega_{l}, \mathbf{q}\right)$, which is essentially the same one as the spin-fluctuation mediated interaction. Experimentally, the exchange interaction is as large as or a little smaller than the bandwidth of quasiparticles. Therefore, high- $T_{c}$ supercon- ductivity of cuprate oxides must occur in the attractive intermediate-coupling regime for superconductivity, which is realized in the repulsive strong-coupling regime for electron correlations, that is, in the vicinity of the Mott metalinsulator transition or crossover.
*Electronic address: fohkawa@phys.sci.hokudai.ac.jp

${ }^{1}$ J. G. Bednortz and K. A. Müller, Z. Phys. B: Condens. Matter 64, 189 (1986).

${ }^{2}$ H. Yasuoka, T. Imai, and T. Shimizu, Strong Correlation and Superconductivity, Springer Series in Solid State Science, Vol. 89 (Springer-Verlag, Berlin, New York, 1989), p. 254.

${ }^{3}$ H. Ding, T. Yokota, J. C. Campuzano, T. Takahashi, M. Randeria, M. R. Norman, T. Mochiku, K. Kadowaki, and J. Giapinzakis, Nature (London) 382, 51 (1996).

${ }^{4}$ J. M. Harris, Z.-X. Shen, P. J. White, D. S. Marshall, M. C. Schabel, J. N. Eckstein, and I. Bozovic, Phys. Rev. B 54, R15665 (1996).

${ }^{5}$ A. G. Loeser, Z.-X. Shen, D. S. Dessau, D. S. Marshall, C. H. Park, P. Fournier, and A. Kapitulnik, Science 273, 325 (1996).

${ }^{6}$ A. Ino, C. Kim, M. Nakamura, T. Yoshida, T. Mizokawa, A. Fujimori, Z.-X. Shen, T. Kakeshita, H. Eisaki, and S. Uchida, Phys. Rev. B 65, 094504 (2002).

${ }^{7}$ Ch. Renner, R. Revaz, J.-Y. Genoud, and $\varnothing$. Fischer, J. Low Temp. Phys. 105, 1083 (1996).

${ }^{8}$ M. Oda, K. Hoya, R. Kubota, C. Manabe, N. Momono, T. Nakano, and M. Ido, Physica C 281, 135 (1997).

${ }^{9}$ T. Nakano, N. Momono, M. Oda, and M. Ido, J. Phys. Soc. Jpn. 67, 2622 (1998).

${ }^{10}$ T. Ekino, Y. Sezaki, and H. Fujii, Phys. Rev. B 60, 6916 (1999).

${ }^{11}$ M. Randeria, N. Trivedi, A. Moreo, and R. T. Scalettar, Phys. Rev. Lett. 69, 2001 (1992).

${ }^{12}$ K. Miyake and O. Narikiyo, J. Phys. Soc. Jpn. 63, 3821 (1994).

${ }^{13}$ J. J. Deisz, D. W. Hess, and J. W. Serene, Phys. Rev. Lett. 80, 373 (1998).

${ }^{14}$ J. R. Engelbrecht, A. Nazarenko, M. Randeria, and E. Dagotto, Phys. Rev. B 57, 13406 (1998).

${ }^{15}$ Y. Yanase and K. Yamada, J. Phys. Soc. Jpn. 68, 2999 (1999).

${ }^{16}$ M. Franz and Z. Tesanovic, Phys. Rev. Lett. 87, 257003 (2001).

${ }^{17}$ V. M. Loktev and V. Turkowski, Int. J. Mod. Phys. B 18, 2035 (2004).

${ }^{18}$ P. Monthoux, Phys. Rev. B 70, 144403 (2004).

${ }^{19}$ P. A. Lee, Physica C 408, 5 (2004).

${ }^{20}$ D. K. Sunko and S. Barisic, Physica C 408, 262 (2004).

${ }^{21}$ A. Ramsak and P. Prelovsek, Physica C 408, 279 (2004).

${ }^{22}$ I. Mamedov, Physica C 408, 341 (2004).

${ }^{23}$ P. A. Marchetti, G. Orso, Z. B. Su, and L. Yu, Physica C 408, 412 (2004).

${ }^{24}$ K. Mitsen and O. Ivanenko, Physica C 408, 422 (2004).

${ }^{25}$ D. Rohe and W. Metzner, Phys. Rev. B 71, 115116 (2005).

${ }^{26}$ Z. C. Gu, T. Li, and Z. Y. Weng, Phys. Rev. B 71, 064502 (2005).

${ }^{27}$ A. A. Katani and A. P. Kampf, Physica B 359, 557 (2005).

${ }^{28}$ N. D. Mermin and H. Wagner, Phys. Rev. Lett. 17, 1133 (1966).

${ }^{29}$ F. J. Ohkawa, Phys. Rev. B 69, 104502 (2004).

${ }^{30}$ The homogeneous susceptibility $\chi_{\Gamma \Gamma^{\prime}}\left(i \omega_{l}, \mathbf{q}=0\right)$ vanishes when the representations of $\Gamma$ and $\Gamma^{\prime}$ waves are different from each other.
${ }^{31}$ If the self-consistency is completed for a repulsive model that is relevant for cuprate-oxide superconductors, the renormalization of the effective attractive interaction, which is discussed in the Appendix, must also be included in the self-consistent procedure.

${ }^{32}$ In the random-phase approximation, where the reduction of $T_{c}$ due to SC critical fluctuations is not considered, it follows that $\lambda=1$ for the critical exponent.

${ }^{33}$ In the limit of $\gamma /|t| \rightarrow+0$, the formulation of this paper is reduced to the conventional non-self-consistent scheme, where bare or unrenormalized Green functions are put into internal electron lines in Feynman diagrams. A pseudogap structure is the sharpest in this limit.

${ }^{34}$ F. C. Zhang and T. M. Rice, Phys. Rev. B 37, R3759 (1988).

${ }^{35}$ See, for example, P. W. Anderson, Basic Notions of Condensed Matter Physics, Frontiers in Physics (Benjamin/Cummings, New York, 1984).

${ }^{36}$ J. W. Loram, K. A. Mirza, J. R. Cooper, and W. Y. Liang, Phys. Rev. Lett. 71, 1740 (1993).

${ }^{37}$ J. M. Luttinger and J. C. Ward, Phys. Rev. 118, 1417 (1960).

${ }^{38}$ J. M. Luttinger, Phys. Rev. 119, 1153 (1960).

${ }^{39}$ M. C. Gutzwiller, Phys. Rev. Lett. 10, 159 (1963); Phys. Rev. 134, A923 (1964).

${ }^{40}$ M. C. Gutzwiller, Phys. Rev. 137, A1726 (1965).

${ }^{41}$ J. E. Hirsch, Phys. Rev. Lett. 54, 1317 (1985).

${ }^{42}$ F. J. Ohkawa, Jpn. J. Appl. Phys., Part 2 26, L652 (1987); J. Phys. Soc. Jpn. 56, 2267 (1987).

${ }^{43}$ H. Ott, D. Brawner, and A. Schilling, J. Low Temp. Phys. 99, 251 (1995)

${ }^{44}$ C. W. Chu, J. Supercond. 7, 1 (1994).

${ }^{45}$ R. A. Klemm, AIP Conference Proceedings (AIP, New York, 1993), No. 273, p. 292.

${ }^{46}$ D. Jérome and H. J. Schultz, Adv. Phys. 51, 293 (2002).

${ }^{47}$ T. Hanaguri, C. Lupien, Y. Kohsaka, D.-H. Lee, M. Azuma, M. Takano, H. Takagi, and J. C. Davis, Nature (London) 430, 1001 (2004).

${ }^{48}$ K. McElroy, D.-H. Lee, J. E. Hoffman, K. M. Lang, J. Lee, E. W. Hudson, H. Eisaki, S. Uchida, and J. C. Davis, Phys. Rev. Lett. 94, 197005 (2005).

${ }^{49}$ F. J. Ohkawa, Phys. Rev. B 73, 092506 (2006).

${ }^{50}$ F. J. Ohkawa, J. Phys. Soc. Jpn. 74, 3340 (2005).

${ }^{51}$ Any doubly occupied configuration at a site is excluded in the $t$ $-J$ model. An infinitely large on-site repulsion $U$ enforces this exclusion in the $t$ - $J$-infinite $U$ model.

${ }^{52}$ J. Hubbard, Proc. R. Soc. London, Ser. A 276, 238 (1963).

${ }^{53}$ J. Hubbard, Proc. R. Soc. London, Ser. A 281, 401 (1964).

${ }^{54}$ F. J. Ohkawa, J. Phys. Soc. Jpn. 58, 4156 (1989).

${ }^{55}$ See, for example, F. J. Ohkawa, Phys. Rev. B 65, 174424 (2002).

${ }^{56}$ The superexchange interaction can be ferromagnetic in a multiband model with a strong enough Hund coupling. See, for example, Ref. 55. 
${ }^{57}$ The exchange interaction $J_{Q}\left(i \omega_{l}, \mathbf{q}\right)$ is also antiferromagnetic, when the nesting of the Fermi surface of Gutzwiller's quasiparticles is sharp. It is strongly ferromagnetic in Kanamori's model for itinerant-electron ferromagnetism considered by J. Kanamori, Prog. Theor. Phys. 30, 275 (1963), where a sharp peak exists in one of the band edges of the density of states and the chemical potential lies around the peak.

${ }^{58}$ F. J. Ohkawa, Phys. Rev. B 59, 8930 (1990).

${ }^{59}$ F. J. Ohkawa, Phys. Rev. B 44, 6812 (1991).

${ }^{60}$ F. J. Ohkawa, J. Phys. Soc. Jpn. 60, 3218 (1991).

${ }^{61}$ F. J. Ohkawa, J. Phys. Soc. Jpn. 61, 1615 (1992).

${ }^{62} \mathrm{See}$, for example, T. Moriya, Spin Fluctuations in Itinerant Electron Magnetism, Springer Series in Solid-State Science, Vol. 56 (Springer-Verlag, Berlin, Heidelberg, New York, Tokyo, 1985).

${ }^{63}$ F. J. Ohkawa, J. Phys. Soc. Jpn. 67, 535 (1998).

${ }^{64}$ E. Miyai and F. J. Ohkawa, Phys. Rev. B 61, 1357 (2000).

${ }^{65}$ The SCR theory (Ref. 62) considers itinerant-electron magnets where the density of state is almost constant around the chemical potential and the Fermi surface shows no nesting. The modemode coupling term is responsible for the $\mathrm{CW}$ law of such itinerant-electron magnets (Ref. 62). On the other hand, the exchange interaction $J_{Q}\left(i \omega_{l}, \mathbf{q}\right)$ is responsible for the CW law of ferromagnets (Ref. 64) where the density of state has a sharp peak around the chemical potential and antiferromagnets (Ref. 63) where the Fermi surface shows a sharp nesting.

${ }^{66}$ In the periodic Anderson model, strongly hybridized admixtures of almost localized electrons and conduction electrons are formed into Gutzwiller's quasiparticles or the so-called heavy electrons at $T \lesssim T_{K}$. On the other hand, they are independent compositions at $T \gg T_{K}$. A relevant physical picture for $T \gg T_{K}$ is the coexistence of localized spins and conduction electrons; the Ruderman-Kittel-Kasuya-Yosida (RKKY) exchange interaction arises from the virtual exchange of a pair excitation of conduction electrons, and it works between localized spins. Then, we can argue that a crossover occurs as a function of $T$ in such a way that the exchange interaction $J_{Q}\left(i \omega_{l}, \mathbf{q}\right)$ at $T \lesssim T_{K}$ turns into the RKKY exchange interaction at $T \gg T_{K}$. The RKKY exchange interaction is never relevant at low temperatures such as $T$ $\lesssim T_{K}$.

${ }^{67}$ J. C. Ward, Phys. Rev. 68, 182 (1950).

${ }^{68} \mathrm{See}$, for example, Ref. 50.

${ }^{69}$ Although the two mechanisms are essentially the same as each other, the spin-fluctuation mechanism where only low-energy spin fluctuations, such as those described by a phenomenological spin susceptibility similar to Eq. (2.16) or those observed by low-energy neutron inelastic scattering experiments, are considered is physically different from the superexchange-interaction mechanism, where high-energy excitations in spin channels are considered.

${ }^{70}$ W. Metzner and D. Vollhardt, Phys. Rev. Lett. 62, 324 (1989).

${ }^{71}$ A. Georges and G. Kotliar, Phys. Rev. B 45, 6479 (1992).

${ }^{72}$ Y. Kakehashi and P. Fulde, Phys. Rev. B 69, 045101 (2004).

${ }^{73}$ Correctly speaking, leading-order intersite effects in $1 / d$ are Weiss's means fields of CDW and isotopic $s$ wave or BCS superconductivity in attractive on-site $U$ models and those of SDW, ferromagnetism and antiferromagnetism, in repulsive onsite $U$ models. Weiss's mean fields of other orders such as $d \gamma$-wave superconductivity are of higher order in $1 / d$. It can be argued that Weiss's mean fields in the presence of infinitesimally small external fields are responsible for the CW law. The CW law for local-moment magnetism, which is due to the temperature dependence of $1 / \widetilde{\chi}_{s}(0)$, is of leading order in $1 / d$. The CW law for itinerant-electron magnetism due to that of the exchange interaction $J_{Q}\left(i \omega_{l}, \mathbf{q}\right)$ is of leading order in $1 / d$, but the CW law due to that of the mode-mode coupling term is of higher order in $1 / d$.

${ }^{74}$ According to the conventional derivation, the superexchange interaction constant between nearest-neighbor sites is as large as $J_{s}=-4|t|^{2} / U$ for the Hubbard model. This value is significantly reduced when nonzero bandwidths of LHB and UHB are considered in the virtual exchange process of a pair excitation of electrons across LHB and UHB. Therefore, the observed $J_{s}=$ $-0.15 \mathrm{eV}$ cannot be consistently explained within the Hubbard model. 